\title{
Tratamiento endoscópico de una hendidura laríngea posterior: Reporte de un caso y revisión de la literatura
}

\author{
Endoscopic treatment of a laryngeal cleft: Case report and literature review \\ Felipe Fredes C', Ricardo Alarcón G1.
}

\begin{abstract}
RESUMEN
Las hendiduras laríngeas posteriores son anomalías congénitas de la laringe de baja incidencia que comprometen la región interaritenoidea o la lámina cricoidea. En caso de extenderse hasta la tráquea son denominados clefts laringo-tráqueo-esofágicos. Su clínica es inespecífica y debe sospecharse en todo niño con trastorno de deglución y neumonía aspirativa a repetición. A continuación, presentamos un caso de un cleft laríngeo tipo 2 tratado endoscópicamente.
\end{abstract}

Palabras clave: Hendidura laríngea posterior, trastorno deglución, neumonía aspirativa, tratamiento endoscópico.

\begin{abstract}
The posterior laryngeal clefts are congenital anomalies of the larynx of low incidence that comprise the interaritenoid region or the cricoid lamina. In case of extending to the trachea they are called laryngo-tracheo-esophagic clefts. Its clinic is non-specific and should be suspected in any child with swallowing disorder and aspiration pneumonia. We present a case of an endoscopically treated laryngeal cleft type 2.

Key words: Posterior laryngeal cleft, swallowing disorder, aspiration pneumonia, endoscopic treatment.

\section{INTRODUCCIÓN}

y la primera reparación quirúrgica exitosa de esta patología fue publicada en 1955 por Petterson ${ }^{3}$.

Los clefts laríngeos se producen entre la $5^{\mathrm{a}} \mathrm{y}$ $7^{\text {a }}$ semana de desarrollo embrionario producto de una alteración en la fusión de los pliegues tráqueoesofágicos 0 en el cierre del anillo cricoide $0^{4}$. Se clasifican de acuerdo a su extensión, siendo la
\end{abstract}

Las hendiduras laríngeas posteriores 0 clefts laríngeos son anomalías congénitas de la laringe extremadamente raras, correspondiendo a menos del $0,3 \%$ de estos casos ${ }^{1}$. La primera descripción de un cleft laríngeo fue realizada por Richter en $1792^{2}$

\footnotetext{
1 Médico del Servicio de Otorrinolaringología y Cirugía de Cabeza y Cuello, Hospital Guillermo Grant Benavente, Concepción.
} 
clasificación más utilizada la propuesta por Benjamin e Inglis en 1989, la cual divide los clefts en 4 tipos según su extensión distal5. Un cleft tipo 1 corresponde a una separación interaritenoidea supraglótica. En el tipo 2 hay un compromiso parcial de la lámina cricoidea. En el tipo 3 existe un compromiso total de lámina del cricoides y puede extenderse hasta la tráquea cervical. En el tipo 4 el compromiso se extiende hasta la tráquea intratorácica ${ }^{5}$.

Su clínica es inespecífica y se caracteriza por la presencia de un trastorno de deglución y las complicaciones derivadas de éste, como neumonías aspirativas a repetición. Debido a lo anterior, estos pacientes pueden ser evaluados por diversos especialistas como gastroenterólogos, broncopulmonares y otorrinolaringólogos, por lo que requieren un alto índice de sospecha para hacer el diagnóstico ${ }^{6}$. A continuación presentamos un caso clínico correspondiente al primer cleft laríngeo tratado en nuestro hospital, el cual fue resuelto mediante un abordaje endoscópico.

\section{CASO CLÍNICO}

Paciente de 6 meses de edad con antecedente de síndrome de Down, hospitalizado en pediatría por neumonía. Se solicitó evaluación a otorrinolaringología por sospecha de trastorno de deglución por presentar tos al deglutir, dificultad progresiva para alimentarse y retraso pondoestatural, motivo por los cuales requirió instalación de una sonda nasogástrica.

Se realizó una evaluación de deglución asistida por nasofaringolaringofibroscopía, en donde se observan signos de reflujo severo y una imagen sospechosa de una hendidura laríngea posterior. Durante la evaluación se evidencia penetración y aspiración. Dados los hallazgos descritos, se decide realizar una revisión de vía aérea bajo anestesia general e iniciar manejo médico con medidas antirreflujo, alimentación asistida por fonoaudiología con espesantes y uso de inhibidores de la bomba de protones. La revisión fue postergada inicialmente por cursar con cuadro de rotavirus.

A los 7 meses de vida se realizó una revisión de vía aérea bajo anestesia general, se palpó la zona interaritenoidea y se confirmó la presencia de una hendidura laríngea posterior que comprometía parcialmente el cricoides por debajo del nivel de las cuerdas vocales, diagnosticándose un cleft laríngeo tipo 2. No se evidenciaron otras lesiones en vía aérea (Figura 1).

Debido a la persistencia de los síntomas pese al tratamiento médico, se decidió realizar una reparación endoscópica del cleft laríngeo en abril de 2016. Se realizó una incisión con monopolar de la mucosa de los bordes laterales del cleft en toda la extensión de la lesión, logrando una separación

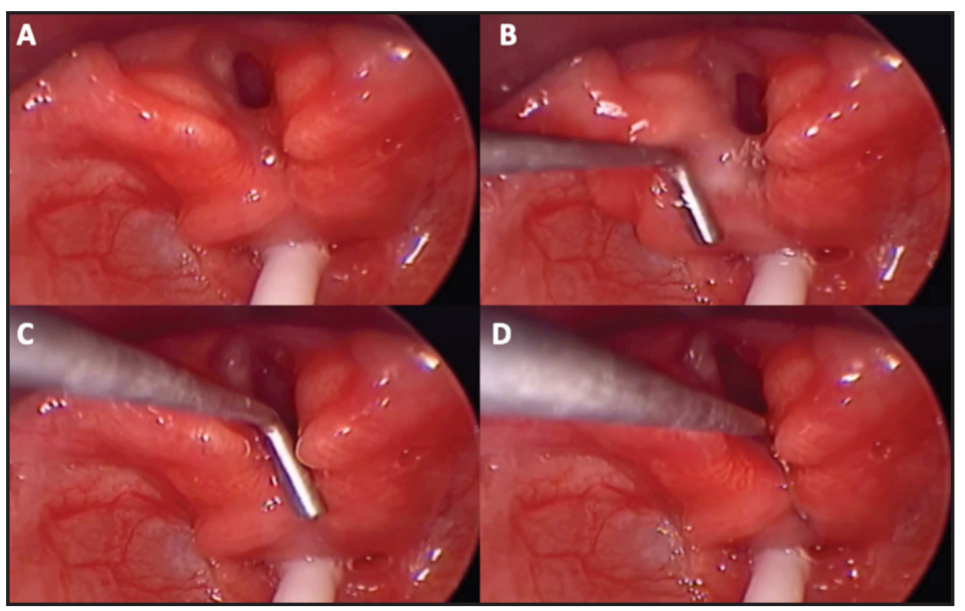

Figura 1. Revisión de vía aérea. (A) y (B) Imagen inicial, se observan burbujas en espacio interaritenoideo lo que hace sospechar la presencia de una hendidura laríngea posterior. (C) y (D) Se palpa la región interaritenoidea confirmando la presencia de un cleft laríngeo que compromete parcialmente el cricoides, por debajo del nivel de las cuerdas vocales. 
adecuada de la mucosa laríngea de la mucosa faringoesofágica. Posteriormente se realizó el cierre del cleft en 2 planos, primero el plano de la mucosa laríngea con vicryl 5.0, desde el extremo distal del cleft hacia proximal, con los nudos hacia la cara faringoesofágica y luego se repite el procedimiento en el plano mucoso faringoesofágico.

En el posoperatorio, persiste con reflujo severo y gran edema de zona operatoria secundario al uso de sonda nasogástrica. En la nasofibroscopía de control se pesquisa recidiva del cleft. Se decidió realizar gastrostomía, optimizar manejo médico y reoperar el cleft.

En noviembre de 2016, se realizó una nueva reparación endoscópica del cleft. En esta ocasión se contaba en nuestro servicio con láser $\mathrm{CO}_{2}$, por lo que la incisión mucosa inicial se realizó con láser, logrando crear una separación adecuada de los dos pliegues mucosos, laríngeo y faringoesofágico, y se procedió a suturarlos desde distal a proximal con vicryl 5.0 de la misma forma antes descrita. Además, se realizó una sección de los pliegues ariepiglóticos para disminuir la tensión en la zona proximal del cleft (Figura 2).

El paciente evolucionó favorablemente, con cicatrización adecuada del sitio quirúrgico, se indicó alimentación mixta por gastrostomía y por boca guiada por fonoaudiología de forma progresiva presentando buena tolerancia. Una revisión de vía aérea realizada en mayo de 2017 evidenció cierre completo del cleft (Figura 3). Actualmente, el paciente recibe alimentación mixta, leche y papilla por gastrostomía y postres por boca, sin presentar tos, con buena tolerancia oral y en cantidades crecientes de comida. No ha vuelto a presentar episodios de neumonía y ha recuperado su curva de crecimiento pondoestatural.

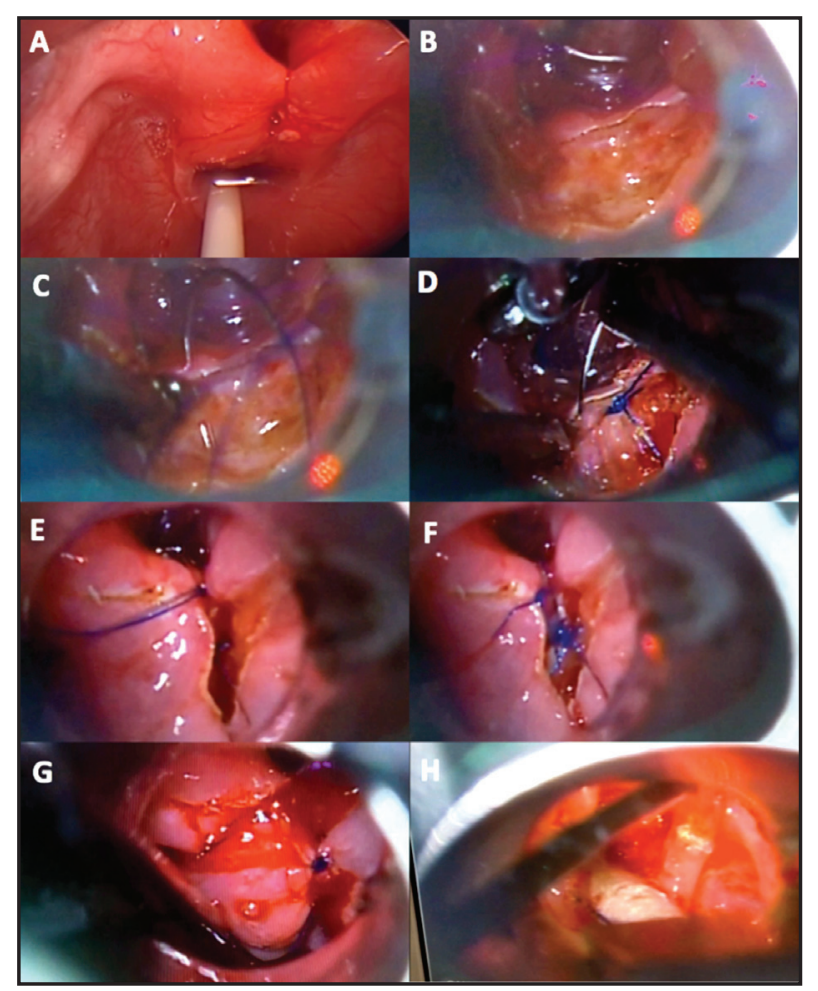

Figura 2. Reparación endoscópica de cleft laríngeo. (A) Cleft laríngeo recidivado. (B) Incisión realizada con láser C02 abarcando toda la extensión del cleft y creando un plano laríngeo y otro faríngeo. (C) Inicio de sutura de plano laríngeo. (D) Continuación de cierre de plano laríngeo, los nudos se dejan hacia la cara faríngea. (E) Cierre completo de plano laríngeo. (F)-(G) Cierre de plano faríngeo. $(\mathrm{H})$ Sección de pliegues ariepiglóticos con láser $\mathrm{CO}_{2}$ para disminuir la tensión en el extremo proximal del cleft. 


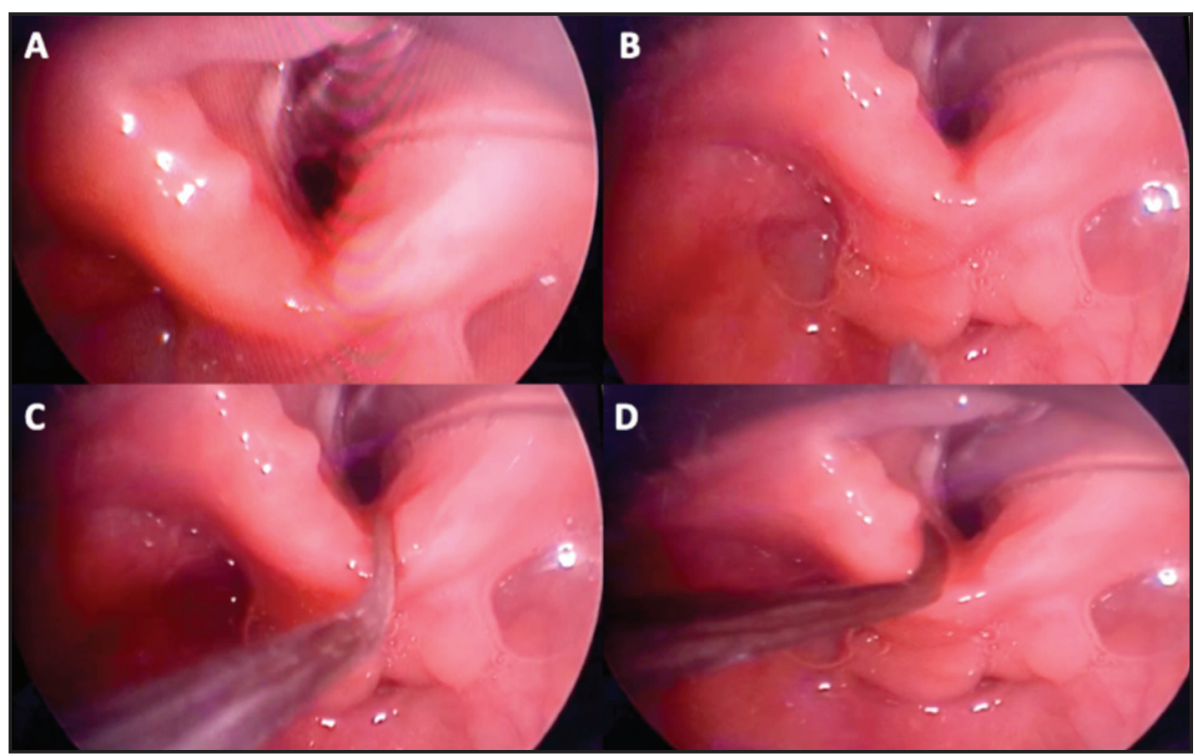

Figura 3. Evaluación posoperatoria. (A)-(B) Se observa la pared laríngea posterior sana, sin hallazgos sugerentes de persistencia de la hendidura posterior. (C)-(D) Se palpa la pared posterior laríngea comprobando el cierre del cleft.

\section{DISCUSIÓN}

Las anomalías laríngeas son alteraciones poco frecuentes con una incidencia estimada de 1 en 2.000 recién nacidos vivos, siendo los clefts laríngeos menos del $0,3 \%$ del total de los casos con una incidencia estimada de 1 cada 10.000 a 20.000 nacidos vivos y una predominancia del sexo masculino (relación hombre:mujer de 5:3)1. El primer caso fue descrito por Richter tras realizar una palpación digital en un lactante que se estaba asfixiando². En 1955 Patterson publicó el primer caso con tratamiento quirúrgico exitoso ${ }^{3}$. Desde esa fecha a la actualidad, se han publicado varias series de casos con incidencias más altas que varían del $6,2 \%$ al $7,6 \%$ de los pacientes con anomalías laríngeas ${ }^{7-9}$. Este aumento en la incidencia se debe a la mayor sospecha de la patología y al mejoramiento en el estudio preoperatorio de estos pacientes mediante laringoscopía directa y palpación de la pared posterior de la laringe bajo anestesia general ${ }^{10}$. Sin embargo, estos estudios son realizados en centros de derivación terciaria especialistas en patología aerodigestiva superior ${ }^{10}$.
Los clefts laríngeos son anomalías congénitas producidas por una falta de desarrollo del músculo interaritenoideo (cleft tipo 1) 0 alteración en el cierre del anillo cricoideo (cleft tipo 2) o en la fusión de los pliegues tráqueo-esofágicos (cleft tipo 3 y 4), produciendo hendiduras de la pared posterior de la laringe de extensión variable ${ }^{4}$. Suelen acompañarse de otras anomalías congénitas en el $58 \%-68 \%$ de los casos, siendo las más frecuentes las gastrointestinales (16\%-67\%), genitourinarias $(14 \%-44 \%)$ y cardiovasculares $(16 \%-33 \%)^{11,12}$. Pueden ser parte de síndromes genéticos como el síndrome de Pallister-Hall y Opitz-Frias principalmente $^{12}$. Otras asociaciones conocidas, pero menos frecuentes, son el síndrome de Down, Vacterl y Charge $\mathrm{e}^{11,12}$.

Como se mencionó previamente, la clasificación más utilizada es la de Benjamin-Inglis la cual los divide en 4 tipos según su extensión ${ }^{5}$. Esta clasificación fue modificada el año 2006 por Sandu y Monnier ${ }^{13}$. Esta clasificación también divide los clefts laríngeos en 4 tipos, pero subdivide los tipos 3 y 4 en dos subtipos. Un cleft tipo 1 es aquel que se extiende por el espacio interaritenoideo por falta del músculo interaritenoideo. El tipo 2 corresponde 
al compromiso parcial de la lámina del cricoides por debajo del nivel de las cuerdas vocales. El tipo 3 compromete la lámina del cricoides en toda su extensión mientras que el tipo 3b se extiende hasta la tráquea cervical. El tipo 4a se extiende a la tráquea intratorácica hasta la carina y el tipo $4 \mathrm{~b}$ hasta comprometer un bronquio fuente ${ }^{13}$.

Su clínica es inespecífica y requiere de un alto grado de sospecha para su diagnóstico en todo niño con problemas para alimentarse y síntomas respiratorios $^{6}$. Las manifestaciones más comunes son: episodios de asfixia durante la alimentación, disfagia con líquidos, estridor, tos crónica, disfonía y neumonías recurrentes ${ }^{6,13}$. El diagnóstico diferencial debe incluir la estenosis esofágica, fístula tráqueo-esofágica, espasmo cricofaríngeo, enfermedades neuromusculares, reflujo gastroesofágico, laringomalacia y parálisis cordal ${ }^{11}$.

El estudio preoperatorio debe estar dirigido en objetivar el trastorno de deglución, determinar el daño pulmonar y demostrar la presencia del cleft. Para esto la evaluación sugerida consiste en la realización de una radiografía de tórax, estudio de deglución mediante videofluoroscopía o test de deglución guiado por endoscopía flexible, y finalmente, la laringoscopía directa bajo anestesia general con palpación del área interaritenoidea y pared posterior laríngea, siendo éste el gold standard actual ${ }^{14}$.

El tratamiento inicialmente es conservador y su objetivo es lograr una nutrición adecuada y prevenir las complicaciones respiratorias. Se basa en aumentar la consistencia de los líquidos y de la comida, el manejo agresivo de las comorbilidades, especialmente del reflujo gastroesofágico ${ }^{14}$. Se reporta una tasa de éxito que varía del $20 \%$ al $100 \% \%^{6-11,15-17}$. Chien y col presentaron una serie de 20 pacientes, en los cuales el tratamiento médico

\section{BIBLIOGRAFÍA}

1. Roth B, Rose KG, Benz-Bohm G, Gunther H. Laryngotracheoesophageal cleft: clinical features, diagnosis and therapy. Eur $J$ Pediatr 1983; 140: 41-6.

2. Richter CF. Dissertatio médico de infanticide inartis obstetriciae. Thesis for Doctor of Medicine. Leipzig, 1792. resultó exitoso solo en el $20 \%{ }^{9}$. Al contrario, Parsons y col. en su serie de 44 pacientes, lograron tratar exitosamente al $100 \%$ de sus casos ${ }^{7}$.

El tratamiento quirúrgico está indicado cuando falla el tratamiento médico y en el caso del cleft tipo 1, el tratamiento endoscópico se ha convertido en el tratamiento de elección ${ }^{18}$. El primer abordaje endoscópico fue realizado por Yamashita en $1979^{19}$. Se considera un abordaje seguro, con una baja morbimortalidad, con una tasa de éxito de $81 \%-94 \%$ 6,8-11,14-16,18,20-23. Es controversial el momento en el cual realizar la cirugía. Day y col en su serie de 18 casos obtuvieron $61 \%$ de éxito en la resolución sintomática al intervenir precozmente a los pacientes antes de 3 meses de realizado el diagnóstico ${ }^{23}$. Rahbar y col propusieron considerar la cirugía cuando el paciente presenta aspiración durante la alimentación, daño pulmonar secundario a la aspiración, alteraciones significativas en la videofluoroscopía y radiografía de tórax, ausencia de otras comorbilidades que generen aspiración, ausencia de otras alteraciones en vía aerodigestiva superior y pobre respuesta al tratamiento médico ${ }^{10}$.

\section{CONCLUSIÓN}

Los clefts laríngeos son una patología poco frecuente, cuya incidencia va en aumento gracias al mayor índice de sospecha y al mejoramiento de las técnicas diagnósticas. Requiere de un manejo multidisciplinario entre el gastroenterólogo, broncopulmonar y otorrinolaringólogo. Es importante recalcar que en todo niño con dificultad para alimentarse y neumonía a repetición se debe sospechar un cleft laríngeo y debe ser sometido a una revisión completa de vía aérea.

3. Pettersson G. Inhibited separation of larynx and upper part of trachea from eosophagus in a newborn: report of a case successfully operated upon. Acta Chir Scand 1955; 110: 250-4.

4. Delahunty JE, Cherry J. Congenital laryngeal clefts. Ann Otol Rhinol Laryngo 1969; 78: 96-106.

5. Benjamin B, Inglis A. Minor congenital laryngeal clefts: diagnosis and classification. Ann Otol Rhinol Laryngol 1989; 98: 417-20. 
6. Ojha S, Ashland Je, Hersh C, Ramakrishna J, Maurer R, HaRTnICK CJ. Type 1 laryngeal cleft: a multidimensional management algorithm. JAMA Otolaryngol Head Neck Surg 2014; 140: 34-40.

7. Parson DS, Stivers FE, Giovanetto DR, Phillips SE. Type 1 posterior laryngeal clefts. Laryngoscope 1998; 108: 403-10.

8. WATTERS K, Russel J. Diagnosis and management of type 1 laryngeal cleft. Int J Pediatr Otorhinolaryngol 2003; 67: 591-6.

9. Chien W, Ashland J, Haver K, Hardy SC, Curren P, HART- NICK CJ. Type 1 laryngeal cleft: establishing a functional diagnostic and management algorithm. Int J Pediatr Oto-rhinolaryngol 2006; 70: 2073-9.

10. Rahbar R, Chen JL, Rosen RL, Lowry KC, Simon DM, Pérez JA, Buonomo C, Ferrari LR, Katz ES. Endoscopic repair of laryngeal cleft type I and type II: when and why? Laryngoscope 2009; 119: 1797-802.

11. Rahbar R, Rouillon I, Roger G, Lin A, Nuss RC, Denoyelle F, McGill TJ, Healy GB, Garabedian EN. The presentation and management of laryngeal cleft: a 10-year experience. Arch Otolaryngol Head Neck Surg 2006; 132: 1335-41.

12. S Pezzettigotta, N Leboulanger, G Roger, F Denoyelle, EN Garabedian. Laryngeal cleft. Otolaryngol Clin North Am 2008; 41: 913-33.

13. Sandu K, Monnier P. Endoscopic laryngotracheal cleft repair without tracheotomy or intubation. Laryngoscope 2006; 116: 630-4.

14. Johnston DR, Watters K, Ferrari LR, Rahbar R. Laryngeal cleft: evaluation and management. Int J Pediatr Otorhinolayngol 2014; 78: 905-11.
15. L.P. Glossop, R.J.H. Smith, J.N.G. Evans. Posterior laryngeal cleft: an analysis of ten cases. Int $J$ Pediatr Otorhinolaryngol 1984; 7: 133-34.

16. H. Kubba, D. Gibson, M. Bailey, B. Hartley. Techniques and outcomes of laryngeal cleft repair: an update to the Great Ormond Street Hospital series. Ann Otol Rhinol Laryngol 2005; 114: 309-13.

17. H.P. van der Doef, J.B. Yntema, F.J. van den Hoogen, H.A. Marres. Clinical aspects of type 1 posterior laryngeal clefts: literature review and a report of 31 patients. Laryngoscope 2007; 117: 859-63.

18. Leishman C, Monnier P, Jaquet Y. Endoscopic repair of laryngotracheoesophageal clefts: experience in 17 cases. Int J Pediatr Otorhinolaryngol 2014; 78: 227-31.

19. M Yamashita, HM Chinyanga, DJ Steward. Posterior laryngeal cleft: anaesthetic experiences. Can Anaesth Soc J 1979; 26: 502-5.

20. Chiang T, McConnell B, Ruiz AG, DeBoer EM, Prager JD. Surgical management of type I and II laryngeal cleft in the pediatric population. Int J Pediatr Otorhinolaryngol 2014; 78: 2244-9.

21. Slonimsky G, Carmel E, Drendel M, Lipschitz N, WoLf M. Type I-II laryngeal cleft clinical course and outcome. Isr Med Assoc J 2015; 17: 231-3.

22. Alexander NS, Liu JZ, Bhushan B, Holinger LD, Schroeder JW JR. Postoperative observation of children after endoscopic type 1 posterior laryngeal cleft repair. Otolaryngol Head Neck Surg 2015; 152: 153-8.

23. Day KE, Smith NJ, KulbeRSH BD. Early surgical intervention in type I laryngeal cleft. Int J Pediatr Otorhinolaryngol 2016; 90: 236-40. 